\title{
Intermédialités
}

Histoire et théorie des arts, des lettres et des techniques

Intermediality

History and Theory of the Arts, Literature and Technologies

\section{Le temps logé en la photographie. À partir de Barthes et Kracauer}

\section{Arlette Farge}

Numéro 7, printemps 2006

URI : https://id.erudit.org/iderudit/1005525ar

DOI : https://doi.org/10.7202/1005525ar

Aller au sommaire du numéro

Éditeur(s)

Centre de recherche sur l'intermédialité

ISSN

1705-8546 (imprimé)

1920-3136 (numérique)

Découvrir la revue

Citer cet article

Farge, A. (2006). Le temps logé en la photographie. À partir de Barthes et Kracauer. Intermédialités / Intermediality, (7), 205-213.

https://doi.org/10.7202/1005525ar
Résumé de l'article

Dans des photos du XX ${ }^{\mathrm{e}}$ siècle que le $\mathrm{XVIII}^{\mathrm{e}}$ siècle ignorait évidemment, quelque chose comme une sédimentation du passé se dépose. Nos corps, nos gestes, comme nos paysages sont emplis de présence d'autrefois dont nous ne sommes pas forcément conscients. Le battement du temps se loge en la photographie. 


\title{
Le temps logé en la photographie. $\grave{A}$ partir de Barthes et Kracauer
}

\author{
Arlette Farge
}

e fugitif, le négligé, l'oublié, le peu visible, l'infra-intersticiel ont toujours

- été pour un parcours d'historien un lieu d'attention. «Lieu » n'est pas le bon mot, plutôt un espace et un mouvement où interagissent l'homme, la femme, ses fragments de réalité, ses correspondances muettes avec autrui et le groupe social. L'histoire officielle ne s'en est guère chargée, trop occupée des faits, des événements et de la continuité temporelle. Pourtant, derrière la continuité se logent les infinies et infimes ruptures, les écarts et les décalages, tout ce qui parfois n’a pas de nom et a cependant une histoire. Le dit puis le non-dit, l'inachevé, le silence sont des objets historiques où se mettent en place par fragments d'éblouissantes apparitions du réel, dans une force politique à reconstituer qui se double d'une esthétique à énoncer.

L'histoire n'est pas si visible qu'on le pense; en tout cas l'invisible histoire est un des pôles majeurs de son socle où les uns se reconnaissent, les autres s'ignorent, fabriquant un tissu dont le contenu peut avoir la force des démissions, des soumissions, des intolérances, mais aussi des espoirs et des révolutions. Décrire, aligner, inventorier, compter n'est pas un but en soi, mais bien plutôt déchiffrer, énoncer, transmettre du sens et du vivant là où on croit que la mort se loge, ou que le temps est fini.

C'est dans cette perspective qu'il m'est arrivé d'accomplir un parcours d'historienne du XVIII ${ }^{\mathrm{e}}$ siècle s'accompagnant de photographies d'aujourd'hui. Et c'est de cette étrange relation que j'essaye de rendre compte. Ce voyage n'avait rien de très naturel, ni de très évident. Plutôt paradoxal. Une première chose à souligner: ce parcours s'est accompli pour moi sans stratégie particulière, et même sans recherche théorique. À part mon goût pour la photographie, aucune lecture savante n’accompagna ce parcours pour en garder l'évidence, la nécessité, et 
peut-être même l'utopie ${ }^{1}$. Ainsi, des photographies d'un passé pas forcément lointain (Valérie Jouve, Lewis Hine, Raymond Depardon, Sophie Ristelhueber, etc.) m'ont fait regarder puis écrire sur des traces du $\mathrm{XVIII}^{\mathrm{e}}$ siècle qui me semblaient enfouies et révélées par les photos que je regardais. Il fallait pour cela le concours d'une écriture se plaçant entre rigueur historique (un savoir profond sur le XVIII ${ }^{\mathrm{e}}$ ), esthétique et contemplation d'une photographie. Il y a donc eu cette tentative d'un passage entre des événements qui sont bien loin d'être contemporains au siècle des Lumières et des figurations, des configurations, des lambeaux de souvenirs, traces, inscriptions du passé, des fragments d'hier venus jusqu'à nous à l'aide d'une technique que ne possède pas le XvIII' : la photographie.

Comme l'écrit Siegfried Kracauer dans son long commentaire d'une photographie de grand-mère à crinoline faisant rire ses petits-enfants qui regardent la photo, la photo ici «représente ce qui est absolument passé, mais ce déchet a été un présent ${ }^{2}$ ». Je reprends cette proposition, mais à l'envers: la photographie d'aujourd'hui elle aussi est déjà, au moment où nous la regardons, le témoin d'un passé, d'un déjà fini. Mais, dans ce déjà fini, il y a ce qui fut et peut-être ce qui se tiendra encore en mouvement, un futur qui adviendra.

Aussi ai-je pu sentir que la photo était un battement du temps. Au premier abord, on peut refuser cette définition puisqu'il n'y a guère de battement du temps dans une photo; cette dernière impose à celui qui la regarde une immobilisation. Le temps est arrêté par l'image; or, il y a un avant et un après lorsqu'une photo se loge devant nos yeux. Et la photographie impose une séparation définitive; celui qui la regarde se trouve en un espace instable où il bascule d'un seul coup entre la vision d'un réel qui déjà n'existe plus et le besoin (quasi morbide ou ludique), suscité par l'image, de retenir, de saisir dans ses mains, charnellement, le temps immobilisé. Il y a du deuil et de la mort dans une photo; on est tendu vers elle car elle arrête le mouvement tandis que notre esprit se prend à le continuer.

Mais la photo suscite aussi du désir: que «cela» recommence un jour comme «cela» fut un jour. Dans cette séparation opère le battement du temps : le geste, l'arbre, le paysage, l'épaule nue, la rue sont immobilisés autant qu'ils sont empreints d'un mouvement qui soudain s'est figé et qui eut lieu, laissant trace en notre corps, tout en retenant encore ce qui se passa en amont de lui.

1. Roland Barthes, La chambre claire. Note sur la photographie, Paris, Éditions des Cahiers du cinéma, Éditions du Seuil, Éditions Gallimard, 1980.

2. Siegfried Kracauer, «La photographie» [1927], dans Le voyage et la danse. Figures de ville et vues de films, trad. Sabine Cornille, Philippe Despoix (éd.), Saint-Denis, Presses universitaires de Vincennes, 1996, p. 42 sq. 
Quelque chose du temps d'avant insiste pour être reconnu et, par traces à peine perceptibles, le temps passé (celui qui ne reviendra pas) recouvre les images du temps d'aujourd'hui. À travers la vision d'une chambre, d'un homme, d'un groupe, d'un ruisseau, d'un membre ou d'un corps enfantin, la photographie offre l'inouïe possibilité de présenter simultanément ce qui y est déposé, ce qui est et ce qui ne figure pas; ce qui existe quelques minutes auparavant dans un ordre sûrement différent, ou quelques siècles avant, et dont on perçoit la griffe, l'infime et résistante trace.

Un peu comme nous nous savons bien être chargés d'une histoire dont nous n'avons pas le souvenir, peuvent se lire ici les marques d'un obstiné passé. La photo est passage. S’y trouve simultanément suggéré le déjà advenu, ce qui advient, ce qui peut-être surviendra. Le battement du temps est ici, embusqué dans la photo: quelque chose des siècles passés y tient son rythme.

Pour que me vienne cette sensibilité particulière à l'instantané photographique, il faut dire quelques mots du travail d'historienne entrepris depuis longtemps. Nul doute qu'il me conduisit vers cette approche. Il se trouve que je travaille essentiellement sur les archives de police du XVIII ${ }^{\mathrm{e}}$ siècle, les mains courantes des commissaires de police, les récits minuscules des plaintes pour petits faits, rixes ou incidents de rue. Ces archives manuscrites sont conservées aux Archives nationales de Paris. Chaque archive, chaque témoignage, chaque plainte pour de petites affaires me permet de travailler à travers les paroles du peuple et celles demandées au peuple pour retrouver ce que furent les comportements populaires, les visions du monde, les conflits, affrontements, désirs, bonheurs et tragédies des sujets du roi avant la Révolution.

Chaque liasse d'archives raconte une scène de vie: dispute, vol, désaccord, chute, jalousie, vengeance, etc. Le récit des interrogés est fragmentaire, rapide, mais c'est une sorte d'arrêt sur image, d'arrêt sur vie, d'immobilisation du temps pour dire ce qui s'est passé. Les paroles dites ici (même face au greffier) sont des événements de parole qui font effraction et permettent à l'historien attentif de retrouver les formes du tissu social d'autrefois.

Ainsi, lire une archive de police (travailler comme historienne en l'interprétant), c'est aussi voir, être sollicitée par les sens, les formes décrites, la couleur des vêtements, la place des objets, la description des rues.

Travailler sur ces vies qui se montrent brièvement sous l'éclairage d'un pouvoir qui contraint à dire, à avouer ou à argumenter, c'est aussi voir un geste, un décor, une faïence brisée, un amas de linge et du sang répandu, des visages marqués, une femme blessée et des enfants au travail. Ainsi épandus ou déposés au greffe, les objets et les vêtements ont quelque chose à voir avec les viscères 
du peuple. Énumérés ainsi parce que les uns sont morts, que les autres sont trop pauvres et vendent, ils déplient une scansion, celle du temps de l'urgence, de la perte et du chagrin. Étalés au greffe, misérables et précieux, les objets sont là ; à prononcer leur nom, à les constater disparates et usés en même temps que réunis, on se fond dans un univers où chaque mot rappelle la respiration de l'homme avec eux, son souffle court quand ces derniers viennent à manquer.

On peut dire la même chose des vêtements décrits des cadavres, du signalement des visages des délinquants. Et encore plus des mots racontés: ceux des bonheurs espérés, des attentes déçues, des destins broyés, des chagrins sans retour, des indignations et des cruautés. Des existences sont là que l'histoire doit raconter et qu'elle a du mal à dire, forcée par l'académisme de sa discipline à privilégier les événements, sans savoir y faire entrer les destins singuliers.

Dans l'archive se lit le poids des êtres parlants, ce battement de l'histoire que l'histoire efface sous son récit officiel. Aussi ai-je longtemps cherché d'autres modes de narration que l'histoire officielle ou le roman pour dire ce monde des autres, transmettre, faire connaître, faire (en fait) re-connaître; car il y a là autant d'étrangeté que de familiarité à retrouver ce monde enfoui, enfui, resté là dans l'ombre de nous-mêmes, prêt à resurgir si on le sollicite, non pour l'établissement de ce fameux devoir de mémoire dont on nous parle tant, mais afin que notre présent aménage son futur dans la connaissance de soi et de l'autre qui habite en soi. Donner à voir, à lire, à comprendre ce passé pour mieux être chacun impliqué dans des figures de l'altérité qui sont nôtres, afin de toujours traquer l'humain dans l'inhumaine condition.

Par son métier, l'historien est naturellement sollicité par le passage du temps et son battement auquel il est accoutumé, car il accomplit souvent le passage entre les époques passées et aujourd'hui, conscient qu'il s'empare de ce qui est déjà fini pour l'expliquer, lui donner sens et interprétation, le décrire, mais plus encore le faire vivre, entrant dans le jardin des morts non pour les calmer, mais pour donner sens à ce qu'ils ont fait ou défait. Il lui est indispensable de se soucier de ce que le présent détient en charge du passé et qui conduit encore ses actes, ses pensées, son imaginaire et même sa vision du passé. Nous sommes pétris d'histoire à notre corps défendant, et cette mémoire inscrite en nos cœurs et corps est un des éléments qui conduit nos pensées et oriente nos comportements.

Il se trouve que, par le type d'histoire du XVIII ${ }^{\mathrm{e}}$ siècle auquel je me suis confrontée, mon lien avec l'image ou du moins la visualisation s'est vite imposé. Dans Le boîtier de mélancolie, Denis Roche écrit que le regard n'abuse pas : «c'est le monde qui déborde et il est bourré d'existences comme de choses. » Bourré d'existences qui sont elles-mêmes habitées par les fantômes du passé, et la photo 
les fixe. La photo est «un secrétariat perpétuel de la présence ${ }^{3}$ : de cette phrase je suis convaincue.

Ainsi, le matériau sur lequel je travaille donne d'emblée un rapport très spécifique à la vie d'autrefois, et il sollicite des modes d'interprétation et des modes de narration très particuliers. On ne peut se contenter d'en faire des anecdotes, il faut les introduire dans un système de sens et d'intelligence qui est le travail de l'historien. Travail dont la responsabilité est de transmettre le savoir historique de la façon la plus précise et véridique possible, mais aussi de parvenir à introduire l'importance des vies et des affects humains dans le déroulement des événements.

À côté de ce travail historique, renouvelé à l'heure actuelle dans son mode de récit (voir Paul Ricœur et son désir d'être au plus près des «êtres parlants » selon Jacques Rancière4), les photographies d'aujourd'hui sont venues m'apporter l'idée du passage. Elles se sont en fait imposées à moi. Elles se sont imposées d'elles-mêmes, parce que faisant surgir à leur vision des traces du passé subsistant en elle comme de longues écharpes, et disant - malgré elles - le «encore là » d'autrefois, toujours persistant dans le présent de l'image. Dans certaines d'entre elles, un souvenir du passé dont je n'ai pas le souvenir charnel me revenait d'un affect, d'un imaginaire, d'une situation, d'un geste ou d'un décor du XviII e siècle.

Toujours à propos de la grand-mère photographiée, Kracauer écrivait: « Sous la photographie d'un être humain, son histoire se trouve enfouie sous un manteau de neige 5 .» Cette phrase m’a particulièrement frappée, car sous la plume de Kracauer, elle procède d'un jugement négatif. Kracauer semble vouloir dire que, de la grand-mère, il n'existe rien, si ce n'est ses habits et crinolines, et qu'il est impossible de la lier à un environnement social et collectif qui fut le sien. Or j'entends cette photo de façon inverse: le manteau de neige qui recouvre la grand-mère est cette pluie d'histoire qui la nimbe et fait d'elle ce que nous voyons, non une poupée, mais un être relié à nous par son histoire. C'est ainsi en tout cas que je regarde les photographies d'autrefois et du présent, et ce qui bien sûr provoque le sourire mais aussi l'effroi. Celui-là même que remarque encore Kracauer quand il dit que les enfants qui voient l'image de leur grand-mère ont simultanément

3. Denis Roche, Le boîtier de mélancolie: la photographie en 100 photographies, Paris, Éditions Hazan, 1999, p. 208.

4. Jacques Rancière, Les noms de l'histoire: essai de poétique du savoir, Paris, Éditions du Seuil, coll. «La librairie du xxe siècle», 1992, p. 4.

5. Siegfried Kracauer, «La photographie», p. 46 . 


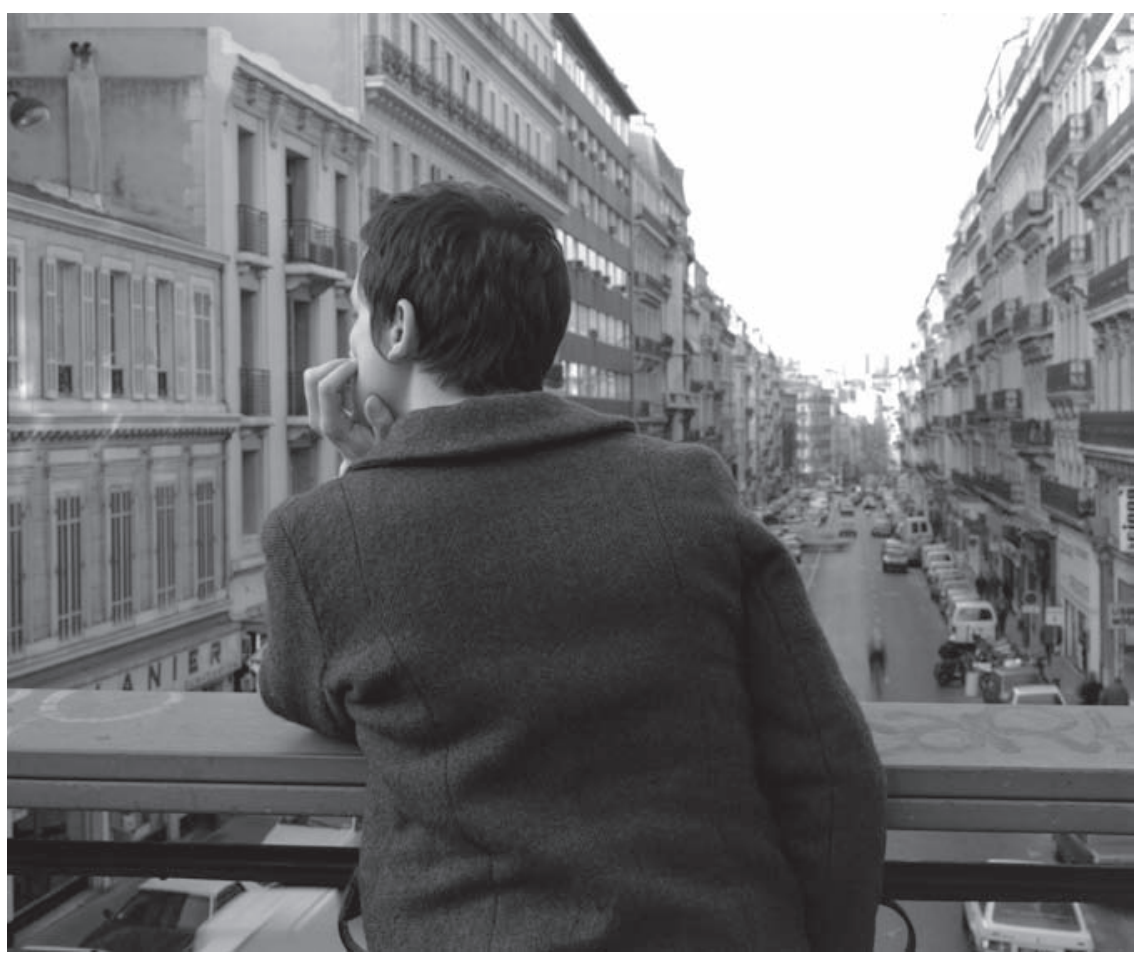

Fig. 1. Valérie Jouve, Sans titre (Les personnages avec Andréa Keen), 1994-1995, photographie couleur, $100 \mathrm{~cm}$ x $130 \mathrm{~cm}$, Galerie Xippas, Paris. (C) Valérie Jouve/SODRAC 2006. Gracieuseté de la Galerie Xippas.

du rire et de l'effroi. Ce n'est pas l'effroi du sans retour, c'est bien sûr l'effroi de la mort, mais aussi l'effroi devant ce qui advient de ce qui est mort.

Je vais prendre rapidement l'exemple de deux photos. La première est celle de Valérie Jouve, Sans titre (Les personnages avec Andréa Keen), (1994-1995, fig. 1), photo qui m’a marquée par sa signifiance historique ${ }^{6}$. Jouve a photographié ce que l'histoire a tant de mal à écrire. C'est une jeune femme de dos sur un balcon surplombant une rue, le visage légèrement penché à gauche et retenu par le creux de la main. Être singulier au cour de l'espace, elle est à elle-même le récit d'une immersion (de surplomb) dans la ville, de l'unique dans le mouvement collectif. Or c'est bien ce qui est le plus difficile à écrire en histoire, tant

6. Arlette Farge, La chambre à deux lits et le cordonnier de Tel-Aviv, Paris, Éditions du Seuil, coll. «Fiction et Cie», 2000, p. 84. 


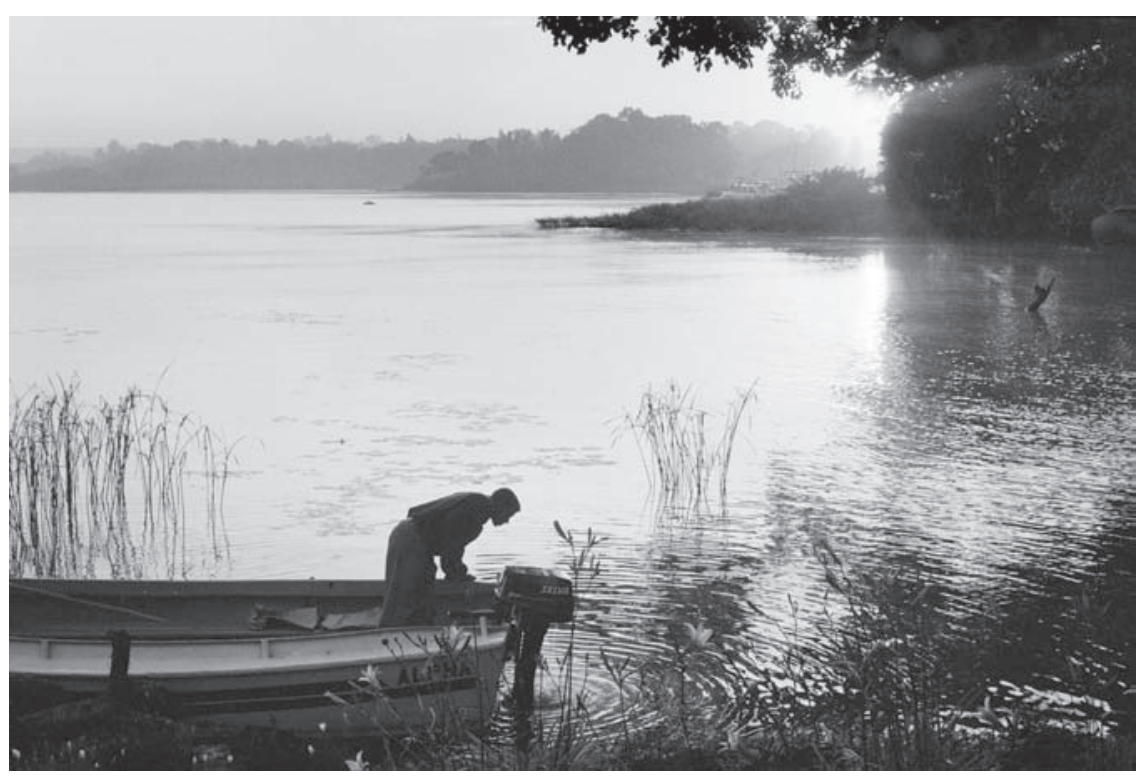

Fig. 2. Raymond Depardon, Éthiopie (Lac Tana, Bahir Dar), 1997, photographie noir et blanc. (C) Magnum Photos.

le récit historique est plus habitué à décrire des comportements collectifs, des mouvements d'ensemble et des visions linéaires d'enchaînements causaux. Comment allier l'être qui vit, aspire, refuse, s'écarte à la série des événements qu'il subit, fabrique, provoque? Ici, le corps est inscrit dans sa résistance à l'espace.

Autre stimulation de cette photo: la présence du corps. Il annonce la force de son inscription dans la réalité du paysage urbain. La prise de conscience passe par une dimension physique du corps immobilisé et mobilisé par l'objectif. Cette photo associe le corps de l'être en train de ressentir à celui qui est modelé par lui. Le corps occupe la ville et, en quelque sorte, la modifie. Cette ville en retour modifie le corps. Corps comme lieu politique de l'inscription sociale. «Nous apprenons par le corps ", écrivait Bourdieu dans ses Méditations pascaliennes, et le corps est exposé, risqué face au monde7 . De sa façon d'être là, d'être contre, d'être avec, il y a moyen de faire histoire, c'est l'image du quelque chose qui tient tête et, en historien, faire le récit sensé de l'enchevêtrement de l'être avec son espace.

7. Pierre Bourdieu, Méditations pascaliennes, Paris, Éditions du Seuil, coll. «Liber», 1997, p. 159. Voir en particulier le chapitre 4, «La connaissance par le corps ». 
La seconde photo est celle de Raymond Depardon, Éthiopie (Lac Tana, Bahir Dar), (1997, fig. 2). Ce paysage d'eau dans sa tranquille douceur, les possibilités de travail qu'il donne, la façon qu'il a d'enfouir un mystère m’a évoqué immédiatement ce sur quoi je suis en train de travailler en histoire: les archives du bord de l'eau autour de Paris au XVIII siècle où vit, se déplace une population importante constamment nomade à l'époque, allant de Paris à la campagne, de la campagne à Paris en suivant fleuves, rivières, cours d'eau. Le XVIII' a connu un paysage aqueux extrêmement fort, modelant les comportements, permettant errances, fuites, passages. Une vie marquée par l'imprévue existait là. Passeurs. Baignades. Rencontres. Délits. Attaques. Les amours furtives. Les noyés et ce qu'ils portent sur eux. Des êtres de précarité dont on retrouve vêtements et objets, leurs écrits sur eux ${ }^{8}$.

La photo du lac paisible et sombre me renvoie à ces temps aussi paisibles que pathétiquement sombres. Dans le silence qu'elle présente, elle n'enfouit pas tant de mystère que cela si l'on veut bien y réfléchir. Elle est l'image aqueuse qui provoque en moi la figure et les dispositions d'hommes et de femmes du $\mathrm{XVIII}^{\mathrm{e}}$ siècle. Sur l'homme au bateau à moteur, sur ses épaules, une histoire existe d'eau et de mouvement, de peine et d'errance. Entre l'une et l'autre je passe et j'écris ce mouvement de va-et-vient.

Ce lieu, ce lien qui s'est tissé naturellement entre la photographie, l'art contemporain et l'histoire du XVIII ${ }^{\mathrm{e}}$ siècle est aussi empreint d'une conviction que les hommes et les femmes sont chargés d'histoire et que celle-ci se révèle encore à leur insu. Par moments, la photo aide, par l'écart direct qu'elle provoque avec le temps, à cette réminiscence et plus que cela : à penser à nouveau cette présence de l'histoire dans les hommes, même si la technique, le progrès, les événements ont changé. Qu'on ne s'y trompe pas, l'histoire qui griffe de ses traces les images d'aujourd'hui ne signifie pas: «Voyez, c'était toujours ainsi, ce sera toujours ainsi. » Je ne crois pas à l'invariance historique, bien au contraire, mais je crois aux façons démultipliées et qui se déclinent à l'infini de l'histoire de l'être insinué en nous sans qu'on en ait la mémoire, et qui nous fait vivre avec elle de façon à la fois très intériorisée et très extérieure à nous9 9 . Dépaysement par rapport à l'histoire et familiarité par rapport à ce qu'elle a pu inscrire en nous se lisent dans certaines photographies. Ainsi les séquences visuelles d'un XVIII ${ }^{\mathrm{e}}$ siècle en train de se faire et de construire le futur imposent son extériorité tout autant que sa radicale intimité avec notre imaginaire d'aujourd'hui. La photo peut être à

8. Arlette Farge, La chambre à deux lits, p. 29.

9. Pierre Laborie, directeur d'études à l'EHESS, m'a souvent expliqué «qu'en histoire ce qu'il y a de prévisible c'est son imprévisibilité ». 
ce point de croisement, repère insolite du « déjà passé » et du «à venir » puisque immobilisé dans l'écart avec le temps.

La photo offre une sorte de pression légère et ferme du lointain sur le proche et du proche reconnu à la lumière du déjà disparu. Aujourd'hui héberge jadis et se logent dans les lisières et les murs les douleurs de mères et la peine des hommes. Regarder une photo, c'est saisir la mort et s'étonner d'elle, voir le vivant et l'accompagner jusque-là.

Barthes écrivait de la photo: «Je voulais l'approfondir, non comme une question, mais comme une blessure: je vois, je sens, donc je remarque, je regarde et je pense $^{10}$.» Blessure, celle du détail qui s'aperçoit furtivement et marque le regard. Blessure, celle du temps qui apporte la douleur. Blessure, celle qui s'ouvre et qui laisse passer dans cette ouverture le lien ténu et profond entre le monde déjà enfoui, enfui et le monde présent. La photo est immobilité dans laquelle s'écrit un vivant frotté et roulé à l'infini dans les infinis passages de sa longue histoire. «Naissance et mort, c'est ce que porte en soi cet instant», et à propos des chemins de fer, Kracauer écrivait: «J'avoue que je n'aime pas terminer un voyage quel qu'il soit ${ }^{11}$. » Je prends cette formule pour mienne.

10. Roland Barthes, La chambre claire, p. 42 .

11. Siegfried Kracauer, «Le chemin de fer » [1930], dans Le voyage et la danse, p. 88. 